\title{
Effects of similarity, difficulty, and nontarget presentation on the time course of visual attention
}

\author{
ROBERT WARD \\ Princeton University, Princeton, New Jersey \\ JOHN DUNCAN \\ MRC Applied Psychology Unit, Cambridge, England \\ and \\ KIMRON SHAPIRO \\ University of Wales, Bangor, Wales
}

\begin{abstract}
Long-lasting interference from an initial visual target on a subsequent one has been measured in two paradigms: rapid serial presentation of targets and nontargets at a single location, and simple presentation of two spatially separated targets. We note that comparisons between these paradigms might be invalid, since interference in each paradigm can be attributed to a different source: demands on selective attention, or demands to switch locations. We use a novel target presentation that both minimizes selection demands and eliminates location switching, yet we still find long-lasting interference. We suggest that all three paradigms discussed tap a common attentional limit. We also examine effects of similarity between targets, and effects of discrimination difficulty on the initial target. We find that similarity effects are more pronounced when nontargets are present, and we find no effect of discrimination difficulty on subsequent interference.
\end{abstract}

Research on the temporal dynamics of visual attention has recently identified an extremely robust pattern of interference, often referred to as the attentional blink (Raymond, Shapiro, \& Arnell, 1992). The typical attentional blink investigation uses rapid serial visual presentation (RSVP) techniques, in which two target items are embedded within a sequence of distractor items, all appearing one after the other in the same spatial location. When the two temporally separated targets must both be processed, identification of the first target ( $\mathrm{T} 1$ ) interferes with processing of the second (T2) for surprisingly long periods, lasting hundreds of milliseconds (Broadbent \& Broadbent, 1987; Raymond et al., 1992; Shapiro, Raymond, \& Arnell, 1994). By comparing T2 performance in conditions where $T 1$ is ignored with performance when $T 1$ is attended, Raymond et al. (1992) ascertained that interference on $\mathrm{T} 2$ is not due to sensory factors such as forward masking from $T 1$, but instead reflects limited-capacity processes associated with attending and responding to a visual target in an RSVP sequence.

Portions of this work were presented in a poster at the 33rd Annual Meeting of the Psychonomic Society, St. Louis. Big thanks to Renee Taylor for running these experiments. Correspondence concerning this article should be addressed to R. Ward, School of Psychology, University of Wales, Bangor, Gwynedd, Wales LL57 2DG (e-mail: r.ward@ bangor.ac.uk).
Similar sustained interference has been found in a spatial analogue of the RSVP task. Duncan, Ward, and Shapiro (1994) measured what they called attentional $d$ well time, using a simple two-target paradigm in which spatially separated targets appeared at varying stimulus onset asynchronies (SOAs), followed by pattern masks. Attention to the first target (T1) produced a pattern of longlasting interference on the identification of the second target (T2), lasting roughly $400 \mathrm{msec}$-comparable to the duration of the attentional blink under RSVP conditions. Other investigators using this simple two-target paradigm have also found long-lasting interference (Moore, Egeth, Berglan, \& Luck, 1996; Ward, Duncan, \& Shapiro, 1996). Thus, both the RSVP and simple two-target paradigms appear to show similar interference resulting from attention to one object on the processing of a subsequent object.

The attractive inference, then, is that both the attentional blink in RSVP and dwell time in the simple two-target paradigm are measures of much the same thing - namely, the time course of interference from one attended object on another. For example, on the basis of results from the simple two-target paradigm, Ward et al. (1996) have outlined a framework in which objects compete in parallel for limited-capacity visual processing resources, such that winners of the competition engage visual processing mechanisms at the expense of losers (a framework that draws heavily from other parallel attention models, such as those of Bundesen, 1990; Duncan, 1980; and Rumel- 
hart, 1970). By this framework, the time course of interference seen in the two-target paradigm is meant to reflect the gradual resolution of the competition for limitedcapacity processing. Shapiro et al. (1994) proposed an account of interference in RSVP based on the Duncan and Humphreys (1989) model, in which target items compete for access to limited-capacity visual short-term memory (VSTM). Chun and Potter (1995) proposed a two-stage model of interference in RSVP, in which a rapid initial detection stage is followed by a slow, limitedcapacity stage necessary for overt response. While an attended item is being processed during the capacity-limited second stage, subsequent items cannot direct overt responses. Thus, similar patterns of interference have been hypothesized to arise from similar sources: the sustained use of limited-capacity resources by attended objects has been used to explain long dwell times in the simple twotarget paradigm (Ward et al., 1996), and attentional blink in the standard RSVP paradigm (Chun \& Potter, 1995; Shapiro et al., 1994).

However, there is a worst case scenario. In the simple two-target paradigm, attention must be reallocated, not only from the first target to the second target, but from the first location to the second location. Interference may not reflect interference of one object on another, but difficulty in reallocating attention from one location to another. In the RSVP paradigm, all items appear at the same location; however, the RSVP stream embeds targets within a series of many irrelevant nontarget items. The RSVP task therefore requires selective processing mechanisms to filter targets from nontargets, a requirement that is minimized in the simple two-target paradigm. Interference in RSVP might reflect difficulties in selection not present in the simple two-target paradigm. Therefore it is possible that the similar patterns of interference found in the two paradigms might be due to different mechanisms: location switching in the two-target paradigm, and rejection of nontargets or other demands of selective processing in RSVP.

To rule out this worst case scenario, we presented a skeletal RSVP stream consisting of only two targets and their respective pattern masks. An alternative, equally appropriate description of our presentation is that we used the simple two-target paradigm, but presented both targets in the same location. This mode of presentation eliminates possible sources of paradigm-specific interference in RSVP and simple two-target presentations. The demands on selective attention in the skeletal RSVP task are the same minimal demands as in the two-target paradigm; the demands for location switching are the same as in RSVP_namely, zero. If the worst case scenario is correct, and RSVP and simple two-target presentation are only tapping these different limitations, our novel mode of presentation should not show extended interference. However, if the two paradigms are tapping a more fundamental, shared attentional limitation-in particular, the interference from one attended object on anotherour skeletal RSVP task should also show a similar, extended pattern of interference.
Besides using a novel mode of target presentation, we also varied the similarity between targets. Similarity between $T 1$ and $T 2$ has been suggested as one important determinant of the attentional blink deficit (Raymond, Shapiro, \& Arnell, 1995; Shapiro et al., 1994). Shapiro et al. (1994) and Raymond et al. (1995) have suggested that similar targets are more likely to interfere with each other than dissimilar ones. Effects of similarity between targets are very marked under conditions of repetition blindness, or RB (Kanwisher, 1987, 1991; Park \& Kanwisher, 1994). In the case of RB, T1 and T2 have the same identity (e.g., both are the letter X). Under these conditions, attention to $\mathrm{T} 1$ results in a profound failure to detect the repeated occurrence at T2 for extended durations of several hundred milliseconds (Park \& Kanwisher, 1994).

Previous results are mixed as to whether RB would be expected with our novel skeletal presentation. Tests of RB have typically used an RSVP or other presentation in which the T1 and T2 items are embedded, either temporally (e.g., Kanwisher, 1987) or spatially (e.g., Kanwisher, 1991; Mozer, 1989), among numerous other items. That is, RB has typically been tested under circumstances more demanding of selective target/nontarget processing than the simple two-target paradigm. When targets are so distinct from nontargets that the result might approximate a simple two-target paradigm - for example, presenting red and green targets against a background of low-contrast black nontargets- RB can be eliminated (Chun, in press, Experiment 3). Recently, however, Kanwisher, Driver, and Machado (1995) found evidence for a form of RB when they used simultaneous presentation of just two spatially separate targets (and subsequent pattern masks). Thus, it seems an open question whether similarity effects will be obtained with our skeletal presentation.

\section{EXPERIMENT 1}

\section{Method}

Subjects. Eight subjects ( 6 female, 2 male; ages from 26 to 41 , mean age 32) were recruited from the Applied Psychology Unit Subject Panel for a single session. The panel is made up of volunteers from the general public, who receive a small honorarium for participation.

Design and Stimuli. Each trial consisted of an RSVP sequence containing only an initial $\mathrm{T} 1$ target item, a subsequent $\mathrm{T} 2$ target separated by a variable SOA, and pattern masks following both these targets (see Figure 1). Items in the RSVP sequence appeared rapidly in the center of a gray screen. In different conditions, subjects attended either to both $\mathrm{T} 1$ and $\mathrm{T} 2$, or just to $\mathrm{T} 2$. To facilitate selective processing of targets, $\mathrm{Tl}$ and its mask were always white, and T2 and its mask were always black. Both targets and masks were presented for a single screen refresh of $15 \mathrm{msec}$, the mask appearing $90 \mathrm{msec}$ after target onset. The SOA between $\mathrm{T} 1$ and $\mathrm{T} 2$ varied as detailed below. The Tl task required subjects to discriminate between two possible sizes for $\mathrm{T} 1$; the $\mathrm{T} 2$ task was to determine whether the second target was the letter $X$.

The experiment was a factorial combination of four factors: similarity (identical or different targets), attention (ignore or attend T1), T2 X presence ( $X$ present or absent), and T1-T2 SOA (eight values, listed below). The similarity factor controlled the identity of $\mathrm{T} 1$ : on identical trials, $\mathrm{T} 1 \mathrm{w}$ was the letter $\mathrm{X}$, on different trials, $\mathrm{Tl}$ was an outline box. (Of course, even in the identical case $\mathrm{T} 1$ and $\mathrm{T} 2$ 


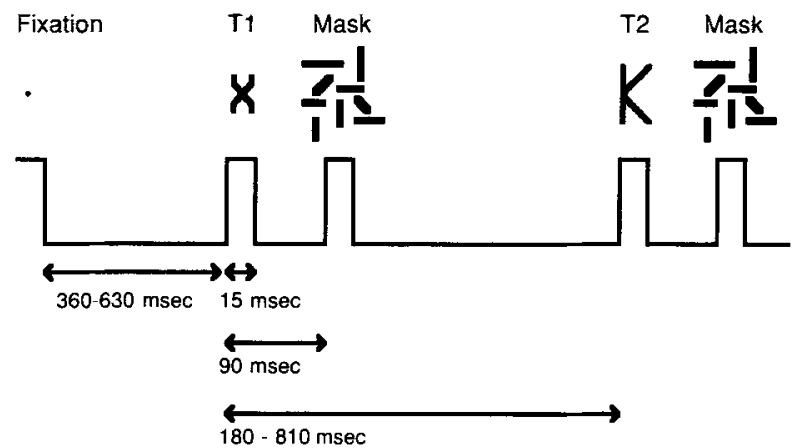

Figure 1. Schematic of a trial in Experiment 1. All items appeared in the center of the display screen. The stimuli depicted (all items shown to scale) are a small T1 and a large T2. Targets and masks were presented for $15 \mathrm{msec}$. Mask latency was 90 msec. Onset asynchrony between $T 1$ and T2 varied from 180 to $\mathbf{8 1 0}$ msec. Following trial initiation and fixation offset was a random delay between 360 and $630 \mathrm{msec}$.

were the same only for the $\mathrm{T} 2 \mathrm{X}$ present trials. $\mathrm{T} 1$ and $\mathrm{T} 2$ were never the same on the $\mathrm{T} 2 \mathrm{X}$ absent trials.) The attention factor determined the task for subjects. In both attend and ignore $\mathrm{T} l$ conditions, subjects were asked to determine whether or not $\mathrm{T} 2$ was an $X$. In the attend $T 1$ conditions, subjects were also asked to report in which of two sizes T1 appeared; in this case, the T1 task was stressed over the T2 task. The T2 X presence and SOA factors controlled the identity and onset of $\mathrm{T} 2$. On $\mathrm{X}$ present trials, $\mathrm{T} 2$ was a black letter $\mathrm{X}$; on $\mathrm{X}$ absent trials, T2 was randomly a black $\mathrm{H}, \mathrm{K}$, or $\mathrm{Y}$. T2 appeared in any one of eight temporal positions, with the SOA following T1 ranging from 180 to $810 \mathrm{msec}$, in $90-\mathrm{msec}$ intervals.

There were three possible sizes for the $\mathrm{T} 2$ item: at a viewing distance of approximately $60 \mathrm{~cm}$, small letters subtended approximately $.24^{\circ} \times .48^{\circ}$, medium letters $.32^{\circ} \times .64^{\circ}$, and large letters $.48^{\circ} \times .96^{\circ}$. The T1 item was of either small or medium size. Pattern masks following T1 and T2 (illustrated in Figure 1) were $1.17^{\circ} \times$ $1.33^{\circ}$.

All factors were varied within subjects. Each block contained 192 trials, 12 trials for each combination of T2 X presence and SOA factors. Within the experimental session, there were four blocks of trials, one for each combination of attention and similarity factors. Order of block presentation was counterbalanced between subjects.

Procedure. Each trial proceeded as follows. A fixation dot was present in the center of the display before the trial began. Subjects initiated the trial by pressing the computer mouse button. At this time, the immediate offset of the fixation dot was accompanied by a $240-\mathrm{msec}$ auditory signal. There was a random delay of $360-$ $630 \mathrm{msec}$ from fixation offset until the presentation of the RSVP sequence. In attend T 1 blocks, subjects were asked to identify the size of the white item (Tl), as well as determine whether the second item (T2) was the letter X. Subjects were instructed to make the size discrimination their first priority. In the ignore $\mathrm{T} 1$ blocks, subjects were asked only to determine whether the second item was the letter $X$. After the sequence was over, the fixation dot appeared again in the center of the display, and subjects made reports verbally. In attend T1 blocks, the T 1 report was made first ("small" or "large"). The T2 report was always "absent" or "present." The experimenter entered responses immediately on a computer keyboard.

Before each block of trials began, subjects were given roughly 20 trials of practice, mainly to provide familiarity with the Tl discrimination required in that block. No feedback was provided during the experiment.

Stimuli were presented on an Apple Hi-Res 13-in. color monitor controlled by an Apple Macintosh IIci running custom software.

\section{Results and Discussion}

Analyses were performed using data from all T2 X present trials. Accuracy on $\mathrm{T} 2 \mathrm{X}$ absent trials was at ceiling (98.7\% over all conditions) and showed no effect of any experimental manipulation. Accuracy on $\mathrm{T} 1$ size judgments averaged $79.6 \%$ correct over all conditions, and we obtained the same pattern of $\mathrm{T} 2$ performance regardless of whether the analysis was conditionalized on T1 accuracy.

Figure 2 presents $\mathrm{T} 2$ accuracy over time, as a function of attention to $\mathrm{T} 1$ and similarity between $\mathrm{T} 1$ and $\mathrm{T} 2$. Two main effects are apparent. First, T2 accuracy is higher in the ignore $\mathrm{T} 1$ than the attend $\mathrm{T} 1$ conditions, averaging $90.2 \%$ and $81.25 \%$, respectively $[F(1,7)=11.03, p=$ $.013]$. Second, there is a main effect of SOA, such that later pusitions are more accurate than early ones $[F(7,49)=$ $10.21, p<.0005]$. These two factors also produced a reliable interaction $[F(7,49)=3.66, p=.003]$, such that accuracy in the attend conditions is initially lower but recovers with time to approximately the same asymptotic levels as in the ignore conditions. These statistics simply confirm the general impression from the figure that attention to $\mathrm{T} 1$ results in extended interference in processing a subsequent $\mathrm{T} 2$.

To estimate the duration of interference from $\mathrm{T} 1$ processing on $\mathrm{T} 2$, we compared accuracy at each SOA in attend and ignore $\mathrm{T} 1$ conditions. T2 accuracy was reliably lower for attend than ignore conditions at SOAs of $450 \mathrm{msec}$ and below $[3.32>t(7)>1.90, .005<p<.05$, one-tailed]. At SOAs greater than $450 \mathrm{msec}$, these differences were not reliable $[t(7)<1.55, p>.05$, one-tailed]. Interference from an attended object on a subsequent object in the present study is sustained for approximately $400-500 \mathrm{msec}$, roughly the same duration as found in

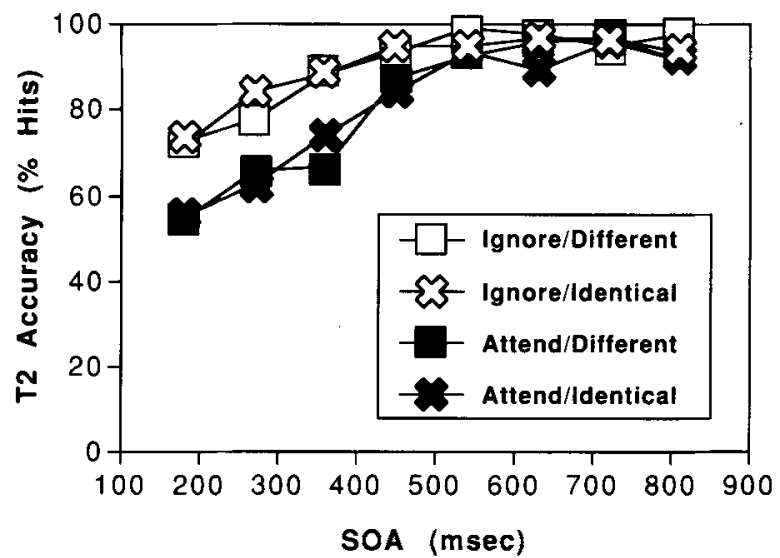

Figure 2. $T 2$ detection accuracy in Experiment 1, no nontargets. Accuracy on $\mathrm{T} 2 \mathrm{X}$ present trials plotted as a function of T1-T2 SOA. In the ignore T1 conditions (open symbols), only $X$ presence or absence for $T 2$ was reported; in attend $T 1$ conditions (filled symbols), the size of $T 1$ was reported in addition to $X$ presence or absence for $\mathrm{T} 2$. In different conditions (square symbols), T1 was a white outline box; in identical conditions (cross symbols), T1 was a white $X$. 
previous studies (both in RSVP, Raymond et al., 1992; Shapiro et al., 1994; and in simple two-target paradigms, Duncan et al., 1994; Ward et al., 1996).

Our results demonstrate substantial interference in the $\mathrm{T} 2 \mathrm{X}$ present trials, but none in T2 X absent trials. A similar pattern of results has been seen before, as reported by Ward et al. (1996, Experiment 4), in which attentional blink interference during a search task was found in target present, but not target absent trials. Ward et al. (1996) suggested that this pattern can be simply explained as a bias in response, such that when there is interference from $\mathrm{T} 1$ on $\mathrm{T} 2$, subjects report that the $\mathrm{T} 2$ target is absent. This response bias account holds equally well for the present study, in which subjects were searching for an $\mathrm{X}$ in the $\mathrm{T} 2$ position. By this account, when subjects are uncertain about $\mathrm{T} 2$ identity, as a result of attentional blink interference or other causes, they make an "absent" response. In this way, blink effects would be manifest only in the T2 X present trials, as in our data.

A simple conclusion from the present study, based on the interference that we have seen with our skeletal presentation, is that the worst case scenario that we described earlier is disconfirmed. Neither selection from a stream of numerous nontargets, nor a shift in attended location, is necessary to produce the extended interference of the attentional blink deficit. It seems likely that under attentional blink conditions, RSVP and simple two-target presentations are both measuring the time course of a common attentional limitation.

Remarkable by its absence, however, is any effect of T1-T2 similarity. Analysis of T2 X present trials revealed no hint of any main effect from identical versus different $\mathrm{T} 1 \mathrm{~s}$ on subsequent interference on $\mathrm{T} 2[85.4 \%$ vs. $86.0 \%$, respectively; $F(1,7)=.13$, n.s.]. Neither was there evidence of any interaction involving similarity; all interaction terms involving similarity produced $F$ values less than 1.0. Thus, repeated T1-T2 pairs were no worse than unrepeated pairs. Although our novel mode of presentation appears to have produced the expected attentional blink deficit, we obtained no effect of similarity.

Several accounts of this null effect of similarity suggest themselves. First, our failure to find an effect of identical T1-T2 pairs might have resulted simply because $\mathrm{T} 1$ identity was completely irrelevant to the tasks in this experiment. Given that the T1 task was to judge the size of the white item, subjects might never have encoded T1 identity, particularly if doing so might later have increased the difficulty of the T2 task. A second possibility is that the null effect of similarity resulted from our use of the skeletal RSVP task, as opposed to the standard RSVP presentation with many nontargets. We will consider these possibilities in more detail in Experiment 2, where we returned to the standard RSVP presentation to look again at effects of $\mathrm{T} 1-\mathrm{T} 2$ similarity. In Experiment 2, we also looked at another variable thought to influence the extent of the attentional blink deficit: difficulty in T1 processing.

\section{EFFECTS OF T1 DIFFICULTY AND MASKING}

It would seem that in attentional blink paradigms, some form of limited capacity is used in processing $\mathrm{T} 1$ and is subsequently unavailable for $\mathrm{T} 2$, and that the blink reflects the availability of this limited capacity over time. What is not clear is the nature of the limited capacity being tapped by $\mathrm{T} 1$ processing. Initial results suggested that the blink was simply not very sensitive to the specifics of T1 processing. For example, Shapiro et al. (1994), in a series of six studies, found no reliable correlation between $\mathrm{T} 1$ performance and the magnitude of the associated blink (as measured by the area between T2 performance curves when $\mathrm{T} 1$ is attended and ignored). For example, the relatively easy T1 task of finding a white $S$ in an RSVP stream of black letters produced much the same magnitude blink as did the relatively hard T1 task of finding a black $S$ in the same stream.

However, recent evidence has disclosed circumstances in which increased difficulty in $\mathrm{Tl}$ processing can produce a larger blink. Chun and Potter (1995) compared the accuracy of letter report for T1 and T2 in an RSVP stream as a function of the item immediately following $\mathrm{T} 1(\mathrm{~T} 1+1)$. In their Experiments 4 and 6, Chun and Potter used either digits or symbol characters (such as an equals sign) in the $\mathrm{T} 1+1$ position. Symbols resulted in both more accurate $\mathrm{T} 1$ report and a reduction of attentional blink deficit, as compared with digits in the $\mathrm{T} 1+1$ position. Chun and Potter suggested that one basis for this difference between symbols and digits could have been their visual characteristics. It is quite possible that digits were a more effective mask for letter stimuli than were some of the other symbols (especially $/,=,<,>$ ). Two other recent studies support this interpretation of Chun and Potter's digit/symbol manipulation. These studies have also examined effects of $\mathrm{T} 1$ difficulty on attentional blink, by manipulating the effectiveness of T1 masking. Seiffert and Di Lollo (in press) argue that effective T1 masking results in larger blinks. For example, they have found that conditions conducive to metacontrast masking both decrease $T 1$ accuracy and increase the size of attentional blink under RSVP conditions. Removing the $\mathrm{T} 1+1$ item entirely, as in Raymond et al. (1992), would also be one way to reduce T1 masking. The effect of removing the $\mathrm{T} 1+1$ item is to eliminate (Raymond et al., 1992) or reduce (Seiffert \& DiLollo, in press) subsequent attentional blink while simultaneously improving T1 performance. Moore et al. (1996) have obtained similar results with the simple two-target paradigm, in which eliminating or delaying the onset of $\mathrm{T} 1$ masking improves $\mathrm{T} 1$ identification and also reduces the magnitude of subsequent interference.

One form of TI difficulty that seems to affect subsequent attentional blink is then the effectiveness of $\mathrm{T} 1$ masking. But what about other forms of $\mathrm{T} 1$ difficulty? Is there a general effect of $\mathrm{T} 1$ difficulty on attentional blink deficits, or do only certain types of $\mathrm{T} 1$ processing affect 
subsequent interference? The answer tells us what sorts of limited-capacity resources are being used during the period of attentional blink deficit. One piece of evidence that might be interpreted in favor of a general T1 difficulty effect could be Seiffert and Di Lollo's extension of the correlation analysis done by Shapiro et al. (1994). Seiffert and Di Lollo increased the power of the analysis by including results from 18 experiments showing significant attentional blink deficits, found in Raymond et al. (1992), Shapiro et al. (1994), Raymond et al. (1995), Chun and Potter (1995), and Seiffert and Di Lollo (in press). This larger database finds a reliable negative correlation $(r=-.73, p<.001)$ between $T 1$ performance and the magnitude of the attentional blink deficit. However, none of the studies in this data set have looked specifically at $\mathrm{T} 1$ difficulty due to factors other than masking, so it is not known to what extent this correlation measures $\mathrm{T} 1$ difficulty specific to masking and $\mathrm{T} 1$ difficulty arising from other sources.

There have yet been no controlled tests of the effect of T1 difficulty, other than masking, on subsequent attentional blink deficit. However, one piece of evidence is suggestive. In a simple two-target paradigm, Ward et al. (1996) asked subjects to identify just the identity, just the size, or both the identity and the size of T1. Size judgments on T1 happened to be reliably easier than identity judgments, but both tasks produced the same degree and duration of interference on T2. Further, concurrent judgments of both size and identity on $\mathrm{T} 1$ produced the same interference, both in duration and degree, as did single judgments of either size or identity. Sustained interference produced by an initial target on a subsequent one is therefore not necessarily sensitive to the number or difficulty of discriminations made to the initial target. In the next experiment, we sought to replicate this finding in a more controlled study, using two degrees of difficulty for a single $\mathrm{T} 1$ discrimination: in this case, an easy and a hard size discrimination.

\section{EXPERIMENT 2}

In this experiment, we looked at effects of T1-T2 similarity and possible interactions with $\mathrm{T} 1$ difficulty in the standard RSVP paradigm. As in Experiment 1, we once again looked at effects of $\mathrm{T} 1-\mathrm{T} 2$ similarity by varying the identity of $\mathrm{T} 1$, while keeping the identity of $\mathrm{T} 2$ constant. We also varied the difficulty of $T 1$ processing by requiring either a difficult or an easy size judgment.

\section{Method}

With the exceptions detailed below, the methods were the same as in Experiment 1.

Subjects. Twelve new subjects were recruited from the Applied Psychology Unit Subject Panel (9 female, 3 male; ages from 21-35, mean age 29).

Design, Stimuli, and Apparatus. Each trial consisted of an RSVP sequence containing an initial white $T 1$ target item, and, on half the trials, a subsequent black T2 item, both embedded in a stream of numerous black nontargets. The T1 task required subjects to discriminate between two possible sizes of the white item; the T2 task required subjects to determine whether or not the letter $\mathrm{X}$ was presented within the sequence.

The RSVP sequence consisted of a pre-T1 stream, T1, and a T2 stream. The pre-T1 stream consisted of $7-15$ nontarget letters. The T2 stream was always 8 letters long: on half of the trials, T2 was absent, and all 8 letters were nontargets; on half, $\mathrm{T} 2$ was present, and the $\mathrm{T} 2$ stream consisted of 7 nontargets and the T2 target (the letter $\mathrm{X}$ ). Nontargets were black uppercase letters selected randomly without replacement from the set of all letters, excluding $S$ and $X$; sizes were selected randomly for each character, using the same small, medium, and large sizes of Experiment 1. Each item in the sequence was presented in the center of the display for a single screen refresh of $15 \mathrm{msec}$, with an SOA of $90 \mathrm{msec}$ between successive items.

The experiment was a factorial design of four factors: similarity (irlentical, same category, different category), T1 difficulty (hard or easy), T2 presence (present or absent), and T1-T2 SOA (eight values specified below). Unlike in Experiment 1, we did not include an attention manipulation, for two reasons. First, it would have necessitated an overly large number of trials; and second, it has been established that ignoring T1 in a standard RSVP stream results in T2 performance relatively unaffected by T1-T2 SOA (see, e.g., Raymond et al., 1992). The appearance of $\mathrm{T} 1$ was constrained by the similarity and difficulty factors, both blocked. There were three possible identities for $\mathrm{T} 1$, as a function of similarity: the letter $\mathrm{X}$ (identical), the letter $\mathrm{S}$ (same category), or an outline box (different category). Possible sizes of T1 were determined by difficulty: in hard blocks, Tl was randomly either small or medium, as in Experiment 1 ; in easy blocks, T1 was either small or large.

The factors of presence and SOA controlled aspects of the T2 stream. On present trials, one of the items in the T2 stream was a black letter X (T2). When present, T2 appeared in any one of eight T1-T2 SOAs ranging from 90 to $720 \mathrm{msec}$, in $90-\mathrm{msec}$ intervals. The size of T2 (small, medium, or large) was determined randomly on each trial.

All factors were varied within subjects as follows. The experiment was divided into two sessions a few days apart. T1 difficulty was varied for each subject between sessions. Within a session, there were three blocks of trials, one for each level of similarity. Each block contained 192 trials: 12 trials for each combination of $\mathrm{T} 2$ presence and SOA factors. Order of presentation for levels of difficulty and similarity were counterbalanced between subjects. Practice was given before each block, as in Experiment 1.

\section{Results and Discussion}

T2 performance on all present trials (see note 1) was subjected to an ANOVA considering the three factors of T1 difficulty, similarity, and SOA. Detection of T2 in the final, unmasked position (SOA $720 \mathrm{msec}$ ) was essentially perfect in all conditions, averaging $99.15 \%$. These trials were excluded from analysis to prevent artifactual interactions with SOA, although including these trials does not alter the pattern of significant results.

Effects of similarity. Figure 3 illustrates the effects of similarity on the attentional blink, plotting T2 performance across time as a function of similarity. Over all conditions, detection of $\mathrm{T} 2$ (the letter $\mathrm{X}$ ) was poorest with the identical T1 $(67.8 \%)$, but there was little difference in $\mathrm{T} 2$ detection whether $\mathrm{T} 1$ was an $\mathrm{S}$ (same category, $81.6 \%$ ) or a box (different category, $84.8 \%$ ). The main effect of similarity was reliable $[F(2,22)=27.24, p<.0005]$. 


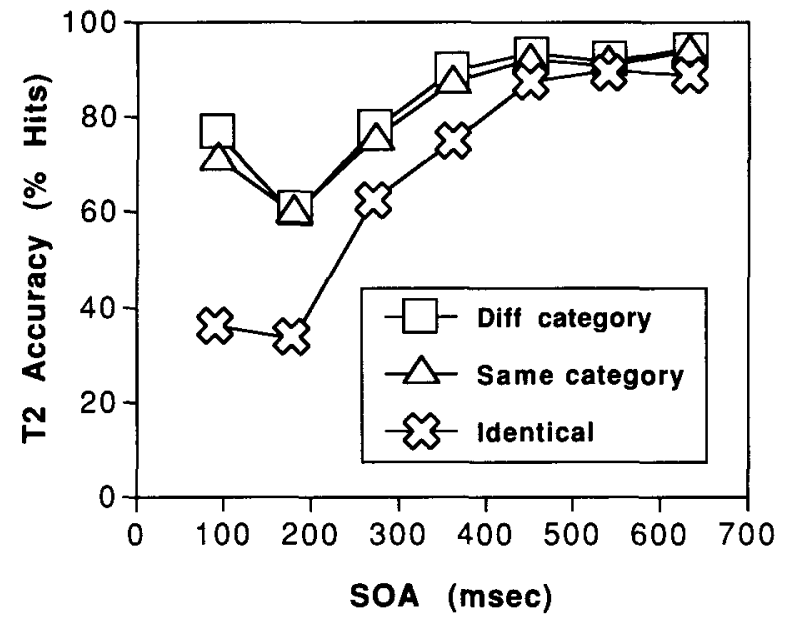

Figure 3. Effects of T1-T2 similarity in Experiment 2, nontargets present. Accuracy on T2 present trials plotted as a function of T1-T2 SOA. Subjects reported both the size of $T 1$ and the presence or absence of $\mathrm{T} 2$. If present, $\mathrm{T} 2$ was always a black letter $X$. The identity of $T 1$ varied as follows: different category conditions (open squares), $\mathrm{T} 1$ was a white outline box; same category (open triangles), $\mathrm{T} 1$ was a white letter S; identical (open crosses), T1 was a white letter $X$.

The interaction of similarity and SOA was also highly reliable $[F(12,132)=7.08, p<.0005]$. The nature of the interaction is apparent from the figure: all levels of similarity show an attentional blink deficit, although the deficit for the identical case is initially deeper, and eventually all conditions return to very similar levels of asymptotic performance. The similarity effect, which was completely absent in Experiment 1 with a skeletal RSVP stream of only two targets, was now clearly evidenced with the standard RSVP presentation of targets embedded among many nontargets.

We estimated the duration of the attentional blink deficit for each level of similarity by comparing performance at each SOA with asymptotic accuracy (defined here as $\mathrm{T} 2$ accuracy at $630 \mathrm{msec}$ ). The results were very similar for all levels of similarity. Between SOAs of 90 and $270 \mathrm{msec}$, accuracy was substantially below asymptote $[t(11)>3.20, p<.005$, one-tailed test]. At the SOA of $360 \mathrm{msec}$, performance was still impaired, but less so $[t(11)>2.32, p<.01]$. At later SOAs performance was not reliably different from asymptotic levels $[t(11)<1.80$, $p>.05]$. Thus similarity affected the magnitude of interference, but with only minimal effects on its duration. The duration of this interference, between 360 and $450 \mathrm{msec}$, was very similar to the duration of interference that we found in Experiment 1.

The effect of similarity on T2 detection is in some ways surprising, since the effect is defined by the identity of $\mathrm{T} 1$, which is, strictly speaking, irrelevant to the T1 task. A post hoc analysis revealed that other irrelevant attributes can modulate interference. We compared T2 performance in conditions where T1 and T2 were of either the same or different sizes. Size was irrelevant to the T2 task. T2 detection was slightly less accurate when $\mathrm{T} 1$ and T2 matched in size than when they did not, but this effect did not reach significance [ $78 \%$ vs. $80 \%$, respectively; $F(1,11)=2.30, p=.157]$. However, size match between $\mathrm{T} 1$ and $\mathrm{T} 2$ did interact marginally with SOA $[F(6,66)=$ $2.29, p=.046]$. This interaction is illustrated in Figure 4, where it can be seen that interference was slightly increased at SOAs between 300 and $500 \mathrm{msec}$ when T1 and $\mathrm{T} 2$ matched in size.

These results extend previous findings on repetition blindness in two ways. First, comparison of Experiments 1 and 2 clearly suggests that RB is more readily obtained when targets are embedded within a presentation of nontarget items. Our findings are consistent with the notion that RB may represent difficulty in token individuation, as suggested by Chun (in press; see also Park \& Kanwisher, 1994). By such an account, salient spatiotemporal discontinuities act as cues to signal the creation of new object tokens. With the nontargets removed in Experiment 1 , the T1 and T2 items could be clearly segmented as distinct spatiotemporal events, providing good evidence for unique $\mathrm{T} 1$ and $\mathrm{T} 2$ tokens, and alleviating $\mathrm{RB}$. With the nontargets reinstated in Experiment 2, the saliency of spatiotemporal cues signaling distinct target events was weakened, so that the $\mathrm{T} 1$ and $\mathrm{T} 2$ events were not as likely to be associated with distinct object tokens; $\mathrm{RB}$ was consequently increased. This account of our RB results also seems compatible with the Shapiro et al. (1994) model of attentional blink, in which the blink results from competition between items in VSTM (also see Raymond et al., 1995). Difficulty in token individuation and token confusion might occur, for instance, in "real time," as items are entering VSTM; or alternatively, "offline," during retrieval of items from VSTM. Of course, this is only one interpretation of our findings. Results from Kanwisher et al. (1995), showing RB under condi-

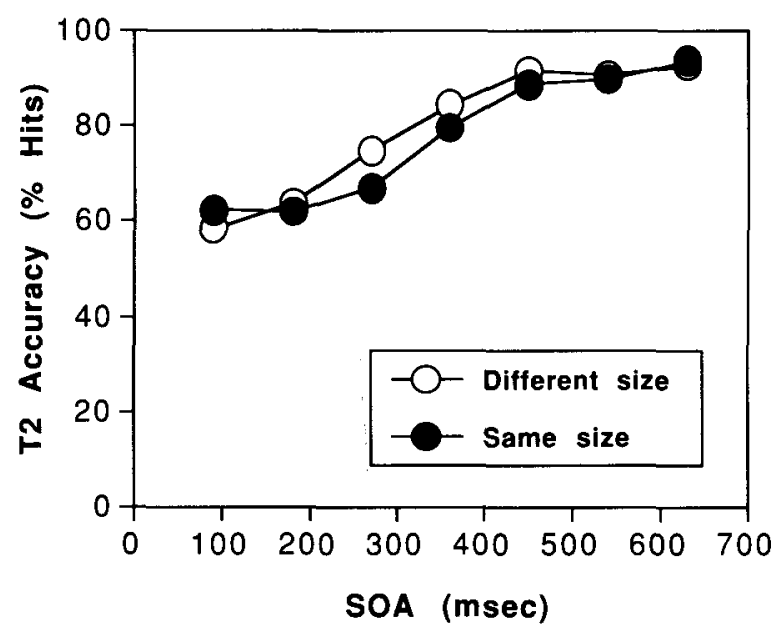

Figure 4. Effects of size match between $T 1$ and $T 2$ in Experiment 2 . Accuracy on $T 2$ present trials plotted as a function of T1-T2 SOA, both when $T 1$ and $T 2$ were different sizes (open circles), and the same size (filled circles). 
tions promoting good segmentation of targets, demonstrate that difficulty in token individuation can be but one cause of RB.

A second extension of previous $R B$ results was found in Experiment 2, where we found circumstances in which RB could arise from irrelevant attributes of T1 and T2. We should add that while we found RB based on irrelevant $T 1$ identity and irrelevant $T 2$ size, neither size nor identity were irrelevant in general (size was relevant for $\mathrm{T} 1$, identity for $\mathrm{T} 2$ ). These results replicate findings by Kanwisher et al. (1995), who also have found RB resulting from irrelevant attributes of attended objects. For example, they found that for reports of the letter identity of $\mathrm{T} 1$ and the color of T2, RB was obtained when T1 and T2 shared color. Kanwisher et al. concluded that there are limits in the selective processing of feature dimensions: when attention selects a particular feature dimension on one object, that same dimension is automatically selected on other objects, at least with the simultaneous presentation used in their experiments. To a large extent, we agree with the Kanwisher et al. conclusions, and extend their results by showing how limits in selective feature processing change with time. At T1-T2 SOAs of less than $450 \mathrm{msec}$, we obtained RB from irrelevant attributes as in Kanwisher et al.; by $450 \mathrm{msec}$, these RB effects were gone. Thus we would amend the Kanwisher et al. conclusions slightly: attention to a dimension of one object may necessitate selection of that dimension on other attended objects for a period of several hundred milliseconds.

Effects of T1 difficulty. As expected, $\mathrm{T} 1$ accuracy was considerably higher with the easy $(98.4 \%$ correct) than with the hard $(84.5 \%$ correct $)$ discriminations $[F(1,11)=$ $100.07, p<.0005]$. In Figure $5, \mathrm{~T} 2$ performance on present trials is plotted as a function of difficulty and SOA. The figure shows the familiar attentional blink function, extended interference following $\mathrm{T} 1$ presentation and iden-

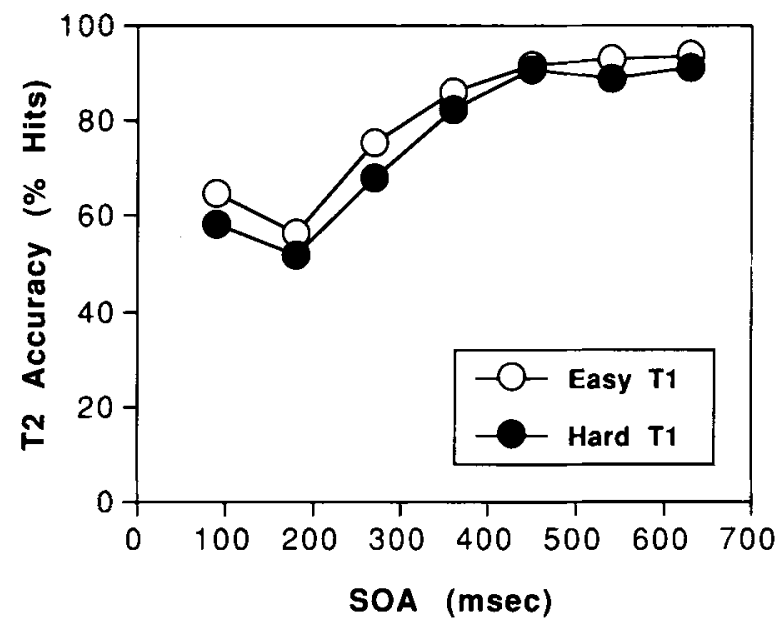

Figure 5. Effects of $\mathrm{T} 1$ size discrimination difficulty in Experiment 2. Accuracy on T2 present trials plotted as a function of T1-T2 SOA. In easy blocks (open circles), T1 could be either small or large in size; in hard blocks (filled circles), T1 was either small or medium in size. tification, lasting roughly $400-500 \mathrm{msec}$ for both easy and hard target discriminations. Despite the large and reliable difference in $\mathrm{T} 1$ performance, difficulty produced no reliable effect on $\mathrm{T} 2$ detection [ $80.2 \%$ on easy trials, $75.9 \%$ on hard; $F(1,11)=2.15, p=.170]$. As suggested by the figure, there was no evidence for any interaction of difficulty and $\operatorname{SOA}[F(6,66)=.597$, n.s. $]$. In fact, there were no reliable interactions of any sort involving difficulty. Further, an ANOVA restricted to the four SOAs below $450 \mathrm{msec}$, where T2 performance was below asymptote, showed no main effect of difficulty on $\mathrm{T} 2$ performance $[F(1,11)=2.42, p=.148]$, and again, in this restricted analysis, no reliable interactions involving difficulty. Finally, separate $t$ tests comparing easy and hard performance at each SOA found no reliable differences [in all cases, $t(11)<1.37, p>.1]$. It appears that difficulties in $\mathrm{T} 1$ discrimination do not necessarily produce the increased attentional blink deficits that have been found with difficulties produced by T1 masking (e.g., as those found by Chun \& Potter, 1995; Moore et al., 1996; Seiffert \& DiLollo, in press).

Is our failure to detect a significant effect of difficulty simply due to lack of power? In our experiment, T1 difficulty was manipulated over a substantial range, with the easy discrimination close to ceiling. The effect produced on T2 accuracy was on the order of .17 standard deviations. Power analysis suggests that such a small effect size would be likely detected only with an enormous sample, roughly 300 subjects. We do not claim there is no effect of $\mathrm{T} 1$ difficulty in this experiment, only that any effect of $\mathrm{T} 1$ discrimination difficulty here is very marginal in comparison with effects that can be attributed to differences in T1 masking efficiency.

Our results with Tl discrimination difficulty demonstrate that not all difficulties in processing $\mathrm{T} 1$ will produce greater subsequent attentional blink. The simple implication is that the attentional blink deficit is not measuring the use of general resources needed for making judgments about object attributes. Given the available evidence, we suggest that masking of $\mathrm{T} 1$ is one factor that does place an additional load on the limited resources reflected in the attentional blink deficit. In the Ward et al. (1996) competitive model of attentional blink, the mask might represent another candidate for target selection and thereby prolong the competition for limited capacity resources, in much the same way as would a second concurrent target (Seiffert \& Di Lollo, in press, Experiment 3; Ward et al., 1996, Experiment 3). Similarly, in the Shapiro et al. (1994) model, the mask may compete with targets for access to VSTM. Chun and Potter (1995), in their twostage model of attentional blink, suggest that visual overlap, which would be consistent with masking, might affect the creation of a durable representation in the relatively slow second stage of their model, necessary for overt report and responses. Their model would account for our results as well, if it is assumed that judgments about targets are made subsequent to the formation of this durable representation. 


\section{CONCLUSION}

In Experiment 1, we used a skeletal RSVP presentation, containing only two targets and their masks, to demonstrate that the long duration of interference from one attended object on another is not dependent on the selection demands of standard RSVP or on the location switching required in the simple two-target paradigm. Surprisingly, with this skeletal presentation, interference was no greater between identical targets than between different targets. In Experiment 2, we used the same tasks as in Experiment 1 but simply reinstated nontargets into the RSVP stream, as in the standard RSVP paradigm. Now the expected repetition blindness effect emerged, so that interference between identical targets was substantially greater than for different targets. In Experiment 2, we also varied the difficulty of discrimination required to the first target and found that hard discriminations produced no more subsequent interference than did easy ones.

Our conclusion is partly methodological. The skeletal RSVP task minimizes demands both on selective attentional processing and on location switching mechanisms. Nevertheless, we still found sustained interference following attention to an object, lasting several hundred milliseconds. We suggest that the sustained interference found with the skeletal RSVP technique confirms that the attentional blink as measured by standard RSVP (Raymond et al., 1992) and dwell time as measured by the simple two-target paradigm (Duncan et al., 1994) are tapping a common attentional limitation. Our worst case scenario, that the RSVP and simple two-target paradigms measure only the time course of two different effects, seems very improbable at this point. However, the choice of paradigm is still important, since we observed effects of target similarity only under standard RSVP conditions. Our point is not entirely methodological, of course. Finding common patterns of interference in three paradigms-standard RSVP, simple two-target presentation, and our skeletal RSVP task - we must ask what the source of this common interference is. In line with recent proposals (Ward et al., 1996), we think it likely that what is measured is the sustained use of limited-capacity resources following attention to an object.

\section{REFERENCES}

Broadbent, D. E., \& Broadbent, M. H. P. (1987). From detection to identification: Response to multiple targets in rapid serial visual presentation. Perception \& Psychophysics, 42, 105-113.

Bundesen, C. (1990). A theory of visual attention. Psychological Review, 97, 523-547.

Chun, M. M. (in press). Types and tokens in visual processing: A dou- ble dissociation between the attentional blink and repetition blindness. Journal of Experimental Psychology: Human Perception \& Performance.

Chun, M. M., \& Potter, M. C. (1995). A two-stage model for multiple target detection in RSVP. Journal of Experimental Psychology. Human Perception \& Performance, 21, 109-127.

DUNCAN, J. (1980). The locus of interference in the perception of simultaneous stimuli. Psychological Review, 87, 272-300.

DUNCAN, J., \& HuMPHREYS, G. W. (1989). Visual search and stimulus similarity. Psychological Review, 96, 433-458.

DUNCAN, J., WARD, R., \& ShaPIRO, K. (1994). Direct measurement of attentional dwell time in human vision. Nature, 369, 313-315.

KANWISHER, N. G. (1987). Repetition blindness: Type recognition without token individuation. Cognition, 27, 117-143.

KANWISHer, N. [G.] (1991). Repetition blindness and illusory conjunctions: Errors in binding visual types with visual tokens. Journal of Experimental Psychology: Human Perception \& Performance, 17, 404-421.

Kanwisher, N. [G.], Driver, J., \& Machado, L. (1995). Spatial repetition blindness is modulated by selective attention to color or shape. Cognitive Psychology, 29, 303-337.

Moore, C. M., Egeth, H., Berglan, L. R., \& Luck, S. J. (1996). Are attentional dwell times inconsistent with serial visual search? Psychonomic Bulletin \& Review, 3, 360-365.

Mozer, M. (1989). Types and tokens in visual letter recognition. Journal of Experimental Psychology: Human Perception \& Performance, 15, 287-303.

PARK, J., \& KANwiSher, N. [G.] (1994). Determinants of repetition blindness. Journal of Experimental Psychology: Human Perception \& Performance, 20, 500-519.

Raymond, J. E., Shapiro, K. L., \& ARNell, K. M. (1992). Temporary suppression of visual processing in an RSVP task: An attentional blink? Journal of Experimental Psychology: Human Perception \& Performance, 18, 849-860.

Raymond, J. E., Shapiro, K. L., \& Arnell, K. M. (1995). Similarity determines the attentional blink. Journal of Experimental Psychology: Human Perception \& Performance, 21, 653-662.

RUMELHART, D. E. (1970). A multicomponent theory of the perception of briefly exposed visual displays. Journal of Mathematical Psy. chology, 7, 191-218.

SEIFFERT, A. E., \& DiLollo, V. (in press). Low-level masking in the attentional blink. Journal of Experimental Psychology: Human Perception \& Performance.

Shapiro, K. L., Raymond, J. E., \& Arnell, K. M. (1994). Attention to visual pattern information produces the attentional blink in RSVP. Joumal of Experimental Psychology: Human Perception \& Performance, 20, 357-371

Ward, R., Duncan, J., \& Shapiro, K. L. (1996). The slow time-course of visual attention. Cognitive Psychology, 30, 79-109.

\section{NOTE}

1. The present design does not allow analysis of absent trials as a function of SOA. T2 performance on absent trials averaged $89 \%$ correct rejections, and T2 correct rejections did not show effects of difficulty [easy, $89.8 \%$; hard, $88.7 \% ; F(1,11)=.399$, n.s.], similarity [different category, $88.5 \%$; same category, $91.6 \%$; identical, $87.6 \% ; F(2,22)=3.16$, $p=.067]$, or a difficulty $\times$ similarity interaction $[F(2,22)=.754$, n.s. $]$

(Manuscript received February 15, 1996; revision accepted for publication July 10, 1996.) 